Pacific Journal of Mathematics

CONDITIONS FOR THE MODULARITY OF AN
ORTHOMODULAR LATTICE 


\title{
CONDITIONS FOR THE MODULARITY OF AN ORTHOMODULAR LATTICE
}

\author{
D. J. Foulis
}

1. Introduction. An orthomodular lattice is a lattice $L$ with 0 and 1 which is equipped with an orthocomplementation ': $L \rightarrow L$ and which satisfies the orthomodular identity $e \leqq f \Rightarrow f=e \vee\left(f \wedge e^{\prime}\right)$. Recall that an orthocomplementation ' $: L \rightarrow L$ maps each element $e \in L$ onto a complement $e^{\prime}$ of $e$ in $L$ in such a way that $e^{\prime \prime}=e$ and $e \leqq f \Rightarrow$ $f^{\prime} \leqq e^{\prime}$ for $e, f \in L$. The "logic" of (non-relativistic) quantum mechanics, i.e., the lattice of closed subspaces of a separable infinite dimensional Hilbert space [5, p. 49], as well as the "logic" of classical mechanics, i.e., the Boolean algebra of all Borel subsets of phase space modulo Borel subsets of measure zero [5, p. 48], are both instances of orthomodular lattices.

L. H. Loomis has shown in [4] that orthomodular lattices provide a natural environment for the abstract study of the dimension theory of operator algebras. I. Kaplansky [3] has obtained an elegant theorem to the effect that if an orthomodular lattice is complete and modular, then it is a continuous geometry.

An involution semigroup is a semigroup $S$ equipped with an involution *, i.e., an antiautomorphism ${ }^{*}: S \rightarrow S$ of period 2 . An element $e \in S$ is called a projection in case $e=e^{*}=e^{2}$. In this paper, we use the term Baer *-semigroup to refer to an involution semigroup $S$ (with a two-sided zero element 0 ) which is equipped with a mapping ' $: S \rightarrow S$ such that

(i) $x^{\prime}$ is a projection for $x \in S$ and

(ii) for $x \in S,\{y \mid y \in s$ and $x y=0\}=x^{\prime} S$. A projection $e \in S$ is said to be closed in case $e=e^{\prime \prime}$, and the collection of all closed projections in $S$ is denoted by $P^{\prime}=P^{\prime}(S)$. The notion of a Baer ${ }^{*}$-semigroup was introduced in [2, §2] in a slightly more general form.

In [2] it is shown that there is an intimate connection between orthomodular lattices and Baer *-semigroups, namely: If $S$ is a Baer *-semigroup, then $P^{\prime}(S)$ is an orthomodular lattice with $e \rightarrow e^{\prime}$ as orthocomplementation and with partial order defined by $e \leqq f \Longleftrightarrow e f$ $=e$ for $e, f \in P^{\prime}(S)$. The element $0^{\prime}=1$ acts as a unit in the semigroup $S$. Conversely, every orthomodular lattice $L$ is isomorphic to a lattice $P^{\prime}(S)$ for some Bear *-semigroup $S$.

In the sequel, the symbol $L$ always denotes an orthomodular lattice and the symbol $S$ always denotes a Baer *-semigroup. When $S$ and $L$ are so related that there is an orthocomplementation preserving iso-

Received September 12, 1960. 
morphism from $P^{\prime}(S)$ onto $L$, we follow [2, §3] by saying that $S$ is a coordinate Baer *-semigroup for $L$. We assume the basic facts on orthomodular lattices and Baer ${ }^{*}$-semigroups as given in $[4$, pp. 3-6] and [2], respectively.

In view of the afore-mentioned result of Kaplansky in [3], and in view of the important role which questions of modularity seem to play in investigations of the "logic" of quantum mechanics [1], it is natural to seek conditions which guarantee that $L$ is modular. The purpose of this paper is to find conditions on coordinatizing Baer *-semigroups $S$ for $L$ which are equivalent to the modularity of $L$. One such condition will be given in terms of the notion of range-closed elements $x \in S$.

Say that $x \in S$ is range-closed in case whenever $g \in P^{\prime}(S)$ with $g \leqq$ $x^{\prime \prime}$ and $\left(g x^{*}\right)^{\prime \prime}=\left(x^{*}\right)^{\prime \prime}$, then $g=x^{\prime \prime} . S$ itself is said to be range-closed in case every element $x \in S$ is range-closed.

As an illustration of the notion of a range-closed element, consider the case in which $S$ is the multiplicative semigroup of all bounded operators on a Hilbert space $H$. Let $*: S \rightarrow S$ be taken, as usual, to mean the passage from an operator $T$ to its adjoint $T^{*}$. Let the operators in $S$ be thought of as operating on the right on the vectors of $H$; and observe that for $A, B \in S, A B=0$ if and only if $B=E B$, where $E$ is the projection onto the orthogonal complement of the range of $A$. Thus, $S$ becomes a Baer *-semigroup if we define ' $: S \rightarrow S$ by $A^{\prime}=$ the projection onto the orthogonal complement of the range of $A$, for every $A \in S$. If $E$ is any projection in $S$, then $(1-E)^{\prime}=E$, hence, $P^{\prime}(S)$ is the lattice of all projections in $S$. Consequently, $P^{\prime}(S)$ is isomorphic to the lattice of all closed linear subspaces of $H$.

If $T \in S$ and if $E \in S$ is the projection onto the closed linear subspace $M$ of $H$, then $\left(E T^{*}\right)^{\prime}$ is the projection onto the closed linear subspace $\left(M^{\perp}\right) T^{-1}$; in particular, $\left(T^{*}\right)^{\prime}$ is the projection onto the null space of $T$. Let $N$ be the range of $T$, let $E$ be a projection in $S$ with $E \leqq T^{\prime \prime}$ = the projection onto the closure of $N$, and let $M$ be the range of $E$. Suppose that $N$ is closed and that $\left(E T^{*}\right)^{\prime \prime}=\left(T^{*}\right)^{\prime \prime}$, so that $\left(M^{\perp}\right) T^{-1}=$ the null space of T. It follows that $M^{\perp} \cap N=0$, i.e., that $E=T^{\prime \prime}$.

On the other hand, if $N$ is not a closed linear subspace of $H$, then $N \neq N^{\perp \perp}$, so there exists a vector $x$ which belongs to $N^{\perp \perp}$ but not to $N$. Let $E_{1}$ be the projection onto the orthogonal complement of the one-dimensional subspace of $H$ spanned by $x$, and let $E=E_{1} \wedge T^{\prime \prime}$. Then, $\left(E T^{*}\right)^{\prime \prime}=\left(T^{*}\right)^{\prime \prime}$, but $E^{\prime} \wedge T^{\prime \prime}=E_{1}^{\prime} \neq 0$; hence $E<T^{\prime \prime}$.

The above argument shows that an operator $T \in S$ is range-closed if and only if the range of $T$ is a closed linear subspace of $H$. Consequently, $S$ is range-closed if and only if $H$ is finite dimensional. Since the lattice of closed linear subspaces of a Hilbert space $H$ is modular if and only if $H$ is finite dimensional, we are led by the above remarks to conjecture that an orthomodular lattice $L$ is modular if and only if 
it can be coordinatized by a range-closed Baer *-semigroup. This conjecture is verified in the sequel.

2. Hemimorphisms of $\mathbf{L}$. In $[2, \S 3]$ we defined a hemimorphism $\phi$ of $L$ to be a mapping $\phi: L \rightarrow L$ such that $0 \phi=0$ and $(e \vee f) \phi=e \phi$ $\vee f \phi$ for $e, f \in L$. We also denoted the semigroup (under function composition) of all monotone maps $\phi: L \rightarrow L$ by $M(L)$, and decreed that two monotone maps $\phi, \psi \in M(L)$ were to be called mutually adjoint in case $(e \phi)^{\prime} \psi \leqq e^{\prime}$ and $(e \psi r)^{\prime} \phi \leqq e^{\prime}$ for all $e \in L$. If $\phi \in M(L)$ has an adjoint in $M(L)$, then this adjoint is unique and is denoted by $\phi^{*} . S(L)$ denotes the subset of $M(L)$ consisting of all those monotone maps $\phi \in M(L)$ which possess adjoints $\phi^{*} \in M(L)$.

We proved in $[2, \S 3]$ that $S(L)$ is a Baer *-semigroup (under function composition), and every $\phi \in S(L)$ is a hemimorphism of $L$. Moreover, if for $e \in L$ we define a mapping $\phi_{e}: L \rightarrow L$ by $f \phi_{e}=\left(f \vee e^{\prime}\right)$ $\wedge e$ for every $f \in L$, then $\phi_{e} \in S(L)$ and $\phi_{e}=\phi_{e}{ }^{*}=\phi_{e}^{2}=\left(\phi_{e}\right)^{\prime \prime}$. The mapping $e \rightarrow \phi_{e}$ is an orthocomplement preserving isomorphism of $L$ onto $P^{\prime}(S(L))$, so $S(L)$ coordinatizes $L$.

In $[2, \S 4]$, we exhibited a natural *-preserving semigroup homomorphism $\phi: S \rightarrow S\left(P^{\prime}(S)\right)$ defined by $x \phi=\phi_{x} \in S\left(P^{\prime}(S)\right)$ for $x \in S$, where $e \phi_{x}=(e x)^{\prime \prime}$ for all $e \in P^{\prime}(S)$. In case $x=f \in P^{\prime}(S)$, there is no notational conflict here; indeed, $(e f)^{\prime \prime}=\left(e \vee f^{\prime}\right) \wedge f$ for all $e \in P^{\prime}(S)$.

Lemma 1. Let $\phi \in S(L), e \in L$. Then, $1 \phi^{*}=\left(e \wedge 1 \phi^{*}\right) \vee e^{\prime} \phi \phi^{*}$.

Proof. Put $g=e^{\prime} \vee\left(1 \phi^{*}\right)^{\prime}, \quad h=g \wedge\left(g \phi \phi^{*}\right)^{\prime} \wedge 1 \phi^{*}$. Since $g \phi \phi^{*} \leqq$ $1 \phi^{*}$, we have $\left(1 \phi^{*}\right)^{\prime} \leqq\left(g \phi \phi^{*}\right)^{\prime}$. Combining the latter inequality with $\left(1 \phi^{*}\right)^{\prime} \leqq g$, we get $\left(1 \phi^{*}\right)^{\prime} \leqq g \wedge\left(f \phi \phi^{*}\right)^{\prime}$; hence, by the orthomodular identity, $g \wedge\left(g \phi \phi^{*}\right)^{\prime}=\left(1 \phi^{*}\right)^{\prime} \vee h$. Now, $g \phi \phi^{*}=e^{\prime} \phi \phi^{*} \vee\left(1 \phi^{*}\right)^{\prime} \phi \phi^{*}=$ $e^{\prime} \phi \phi^{*}$ since $\left(1 \phi^{*}\right)^{\prime} \phi=0$. Consequently, $g \wedge\left(e^{\prime} \phi \phi^{*}\right)^{\prime}=\left(1 \phi^{*}\right)^{\prime} \vee h$, and the lemma will be proved as soon as we show that $h=0$. But, $h \phi \leqq g \phi \wedge$ $\left(g \phi \phi^{*}\right)^{\prime} \phi \leqq g \phi \wedge(g \phi)^{\prime}=0$, so $h \phi=0$. Thus, $1 \phi^{*}=(h \phi)^{\prime} \phi^{*} \leqq h^{\prime}$, so $h \leqq$ $\left(1 \phi^{*}\right)^{\prime}$. Since also $h \leqq 1 \phi^{*}$, it follows that $h=0$, proving the lemma.

THEOREM 2. For $\phi \in S(L)$, the following conditions are equivalent:

(i) $\phi$ is range-closed.

(ii) $\left(f \phi^{*}\right)^{\prime} \phi=f^{\prime} \wedge 1 \phi$ for $f \in L$.

(iii) For $e, f \in L, f \phi^{*}=e \phi^{*} \Rightarrow f \vee(1 \phi)^{\prime}=e \vee(1 \phi)^{\prime}$.

Proof. To prove (i) $\Rightarrow$ (ii), note that $\left(f \phi^{*}\right)^{\prime} \phi \leqq f^{\prime} \wedge 1 \phi$ and put $h^{\prime}$ $=f^{\prime} \wedge 1 \phi \wedge\left[\left(f \phi^{*}\right)^{\prime} \phi\right]^{\prime}$. It will suffice to prove $h^{\prime}=0$. Now, $h \phi^{*}=f \phi^{*}$ $\vee(1 \phi)^{\prime} \phi^{*} \vee\left(f \phi^{*}\right)^{\prime} \phi \phi^{*}=f \phi^{*} \vee\left(f \phi^{*}\right)^{\prime} \phi \phi^{*}=1 \phi^{*}$ by Lemma 1. Since $(1 \phi)^{\prime}$ 
$\leqq h, h=(1 \phi)^{\prime} \vee(h \wedge 1 \phi)$, so $h \phi^{*}=(h \wedge 1 \phi) \phi^{*}$. Thus, we have $(h \wedge$ $1 \phi) \phi^{*}=1 \phi^{*}$. The hypothesis that $\phi$ is range-closed now yields $h \wedge 1 \phi$ $=1 \phi$, so $1 \phi \leqq h$. Consequently, $1 \leqq h$ and $h^{\prime}=0$.

To prove (ii) $\Rightarrow$ (iii), note that according to (ii), $f \phi^{*}=e \phi^{*} \Rightarrow f^{\prime} \wedge 1 \phi$ $=\left(f \phi^{*}\right)^{\prime} \phi=\left(e \phi^{*}\right)^{\prime} \phi=e^{\prime} \vee 1 \phi$. Consequently, $f \phi^{*}=e \phi^{*} \Rightarrow f \vee(1 \phi)^{\prime}=e$ $\vee(1 \phi)^{\prime}$.

To prove (iii) $\Rightarrow$ (i), suppose $g \leqq 1 \phi$ and $g \phi^{*}=1 \phi^{*}$. Then, by (iii), $g \vee(1 \phi)^{\prime}=1 \vee(1 \phi)^{\prime}=1$, so $1 \phi=g \vee\left(g^{\prime} \wedge 1 \phi\right)=g \vee 0=g$.

THeOREm 3. Let $\phi \in S(L)$ be range-closed and let $f \in L, e=(1 \phi)^{\prime}$, $f_{1}=e^{\prime} \phi_{f}$. Then, the necessary and sufficient condition that $\phi \phi_{f}$ fails to be range-closed is the existence of an element $g \in L$ such that $g<f_{1}$ and $g \vee e=f \vee e$.

Proof. By definition, $\phi \phi_{f}$ fails to be range-closed in $S(L)$ if and only if there exists $g<1 \phi \phi_{f}=f_{1}$ such that $g \phi_{f} \phi^{*}=1 \phi_{f} \phi^{*}=f \phi^{*}$. Since $g<f_{1} \leqq f$, we have $g \phi_{f}=g$; hence, $\phi \phi_{f}$ fails to be range-closed if and only if there exists $g<f_{1}$ with $g \phi^{*}=f \phi^{*}$. Because $\phi$ is range-closed, the condition $g \phi^{*}=f \phi^{*}$ is equivalent to $g \vee e=f \vee e$ by part (iii) of Theorem 2.

The hemimorphism $\phi \in S(L)$ will be called totally range-closed in case $\phi_{e} \phi$ is range-closed for every $e \in L$. (In the special case in which $L$ is the lattice of closed subspaces of a Hilbert space $H$, every bounded operator $T$ on $H$ with the property that it maps closed subspaces of $H$ onto closed subspaces of $H$ induces a totally range-closed hemimorphism $\phi_{T}$ on $L$.)

Lemma 4. $\phi \in S(L)$ is totally range-closed if and only if $\left[\left(g \phi^{*}\right)^{\prime}\right.$ $\wedge e] \phi=g^{\prime} \wedge e \phi$ for all $g, e \in L$.

Proof. Let $e \in L$. Then, by part (ii) of Theorem 2, $\phi_{e} \phi$ is rangeclosed if and only if $\left(g \phi^{*} \phi_{e}\right)^{\prime} \phi_{e} \phi=g^{\prime} \wedge e \phi$ for every $g \in L$. It is easy to verify that $\phi_{e}$ is range-closed, so, again by part (ii) of Theorem 2, $\left(g \phi^{*} \phi_{e}\right)^{\prime} \phi_{e}=\left(g \phi^{*}\right)^{\prime} \wedge e$. Hence, $\phi_{e} \phi$ is range-closed if and only if $\left[\left(g \phi^{*}\right)^{\prime}\right.$ $\wedge e] \phi=g^{\prime} \wedge e \phi$ for every $g \in L$.

Denote by $S_{T_{R O}}(L)$ the subset of $S(L)$ consisting of those hemimorphims $\phi \in S(L)$ such that both $\phi$ and $\phi^{*}$ are totally range-closed. Suppose that $\phi$ and $\psi$ are totally range-closed hemimorphisms in $S(L)$. Then, for $g, e \in L,\left[\left(g \psi^{*} \phi^{*}\right)^{\prime} \wedge e\right] \phi \psi=\left[\left(g \psi^{*}\right)^{\prime} \wedge e \phi\right] \psi=g^{\prime} \wedge e \phi \psi ;$ hence, by Lemma $4, \phi \psi$ is totally range-closed. It follows that $S_{r R O}(L)$ is a *-subsemigroup of $S(L)$.

3. *-Regular Baer *-semigroups. Borrowing some terminology from [3, p. 525], we say that $f \in P^{\prime}(S)$ is a right projection for $a \in S$ in case 
$S f=S a$, and we say that $S$ is ${ }^{*}$-regular in case every element $a \in S$ has a right projection. It is plain that $a \in S$ has a right projection $f \in P^{\prime}(S)$ if and only if $f=a^{\prime \prime}$ and $f=b a$ for some $b \in S$.

Now, suppose for a moment that $L$ is complete and modular and that $L$ contains four or more independent perspective elements. By the afore-mentioned theorem of Kaplanksy [3], L is a continuous geometry, and by the well-known coordinatization theorem for continuous geometries, $L$ can be coordinatized by a *-regular ring $R$. If $S$ represents the multiplicative semigroup of $R$, then $S$ is a ${ }^{*}$-regular Baer *-semigroup coordinatizing $L$.

Thus, we are led to a second conjecture: An orthomodular lattice $L$ is modular if and only if it can be coordinatized by $a^{*}$-regular Baer *-semigroup. This conjecture will also be verified in the sequel.

Slight modifications of the proof of [3, Lemma 4, p. 525] give the following lemma:

Lemma 5. Let $a \in S$ have a right projection $f$ and let $a^{*}$ have a right projection $e$. Then, there is a uniquely determined element $a^{-1}$ $\in S$ such that $a^{-1} a=f$ and $a^{-1} e=a^{-1}$. Moreover, $a a^{-1}=e$ and $f a^{-1}$ $=a^{-1}$.

We will follow Kaplansky in [3, p. 525] by calling the element $a^{-1}$ of Lemma 5 the relative inverse of $a$ in $S$. Evidently, $\left(a^{-1}\right)^{-1}=a$ and $\left(a^{*}\right)^{-1}=\left(a^{-1}\right)^{*}$.

THEOREM 6. Let $\phi \in S_{\text {rRo }}(L)$. Then $\phi$ and $\phi^{*}$ both have right projections in $S(L)$ and $\phi^{-1}$, the relative inverse of $\phi$ in $S(L)$, is given by the prescription $g \phi^{-1}=\left[\left(g^{\prime} \wedge 1 \phi\right) \phi^{*}\right]^{\prime} \wedge 1 \phi^{*}$ for $g \in L$.

Proof. Let $e=1 \phi^{*}, f=1 \phi$, and let $\phi^{-1}: L \rightarrow L$ be the mapping given by the prescription of the theorem. For $g \in L, g \phi^{-1} \phi=\left\{\left[\left(g^{\prime} \wedge\right.\right.\right.$ $\left.\left.f) \phi^{*}\right]^{\prime} \wedge e\right\} \phi=\left[\left(g^{\prime} \wedge f\right) \phi^{*}\right]^{\prime} \phi$. Since $\phi \in S_{r R C}(L)$, it is range-closed, so by part (ii) of Theorem 2, $\left[\left(g^{\prime} \wedge f\right) \phi^{*}\right]^{\prime} \phi=\left(g^{\prime} \wedge f\right)^{\prime} \wedge f=g \phi_{f}$. This proves that $\phi^{-1} \phi=\phi_{f}=\phi^{\prime \prime}$. Since, for $g \in L, g \phi^{-1} \leqq e$, we have $g \phi^{-1} \phi_{e}=g \phi^{-1}$; hence, $\phi^{-1}\left(\phi^{*}\right)^{\prime \prime}=\phi^{-1} \phi_{e}=\phi^{-1}$. It only remains to prove that $\phi^{-1} \in S(L)$.

Define $\left(\phi^{-1}\right)^{*}: L \rightarrow L$ by $\left.h\left(\phi^{-1}\right)^{*}=\left[h^{\prime} \wedge e\right) \phi\right]^{\prime} \wedge f$ for $h \in L$. It is plain that $\phi^{-1}$ and $\left(\phi^{-1}\right)^{*}$ are monotone maps on $L$. For $\mathrm{g} \in L,\left(g \phi^{-1}\right)^{\prime}$ $\left(\phi^{-1}\right)^{*}=\left\{\left[\left(\left(g^{\prime} \wedge f\right) \phi^{*}\right)^{\prime} \wedge e\right] \phi\right\}^{\prime} \wedge f=\left[\left(g^{\prime} \wedge f\right)^{\prime} \wedge e \phi\right]^{\prime} \wedge f=\left[\left(g^{\prime} \wedge f\right)^{\prime} \wedge\right.$ $f]^{\prime} \wedge f=\left(g^{\prime} \wedge f\right) \phi_{f}=g^{\prime} \wedge f$. Similarly, for $h \in L,\left[h\left(\phi^{-1}\right)^{*}\right]^{\prime} \phi^{-1}=h^{\prime} \wedge e$; hence, $\phi^{-1}$ and $\left(\phi^{-1}\right)^{*}$ are mutually adjoint and $\phi^{-1} \in S(L)$.

THeOREM 7. Let $L$ be modular. Then, $\phi \in S_{R_{R O}}(L) \Rightarrow \phi^{-1} \in S_{r_{R O}}(L)$.

Proof. Let $g, h \in L$ and let $e=1 \phi^{*}, f=1 \phi, k=\left[\left(h^{\prime} \wedge e\right) \phi\right]^{\prime}$. Since $L$ is modular, $\left((k \wedge f) \vee g^{\prime}\right) \wedge f=(k \wedge f) \vee\left(g^{\prime} \vee f\right)$. Thus, by Theorem 
6, $\left[\left[h\left(\phi^{-1}\right)^{*}\right]^{\prime} \wedge g\right] \phi^{-1}=\left[\left[\left((k \wedge f) \vee g^{\prime}\right) \wedge f\right] \phi^{*}\right]^{\prime} \wedge e=\left[\left[(k \wedge f) \vee\left(g^{\prime} \wedge f\right)\right]\right.$ $\left.\phi^{*}\right]^{\prime} \wedge e=\left[(k \wedge f) \phi^{*} \vee\left(g^{\prime} \wedge f\right) \phi^{*}\right]^{\prime} \wedge e$. Since $\phi^{*}$ is totally range-closed, $(k \wedge f) \phi^{*}=\left(h^{\prime} \wedge e\right)^{\prime} \wedge f \phi^{*}=\left(h^{\prime} \wedge e\right)^{\prime} \wedge e$. Consequently, $\left[\left[h\left(\phi^{-1}\right)^{*}\right]^{\prime} \wedge\right.$ $g] \phi^{-1}=\left[\left(h^{\prime} \wedge e\right) \vee e^{\prime}\right] \wedge\left[\left(g^{\prime} \wedge f\right) \phi^{*}\right]^{\prime} \wedge e=\left(h^{\prime} \wedge e\right) \phi_{e} \wedge g \phi^{-1}=h^{\prime} \wedge e \wedge$ $g \phi^{-1}=h^{\prime} \wedge g \phi^{-1}$, so $\phi^{-1}$ is totally range-closed. A dual argument shows that $\left(\phi^{-1}\right)^{*}$ is also totally range-closed, completing the proof.

LEMmA 8. If $L$ is not modular, there exist elements $e, f, g \in L$ such that $g<e^{\prime} \phi_{f}$ and $g \vee e=f \vee e$.

Proof. If $L$ is not modular, there exist elements $a, b, c \in L$ such that $b<c, b \vee a=c \vee a$ and $b \wedge a=c \wedge a$. Let $h=(b \wedge a)^{\prime}=(c \wedge$ $a)^{\prime}, e=a \phi_{h}, f=c \phi_{h}$ and $g=b \phi_{h}$. Since $h^{\prime} \leqq a, b, c$, we have $e=a \wedge h$, $f=c \wedge h$ and $g=b \wedge h$. Furthermore, since $b \vee a=c \vee a, g \vee e=$ $b \phi_{h} \vee a \phi_{h}=(b \vee a) \phi_{h}=(c \vee a) \phi_{h}=c \phi_{h} \vee a \phi_{h}=f \vee e$. Also, $g=b \wedge h$ $\leqq c \wedge h=f$. If $g=f$, then $b \phi_{h}=c \phi_{h}$, so by part (iii) of Theorem 2 and the fact that $\phi_{h}$ is range-closed we deduce $b \vee h^{\prime}=c \vee h^{\prime}$, i.e., $b=c$, contradicting $b<c$. Thus, we have $g<f$. Finally, $e \wedge f=a \wedge c \wedge h$ $=0$, so $f=(e \wedge f)^{\prime} \wedge f=e^{\prime} \phi_{f}$, completing the proof.

THEOREM 9. L is modular if and only if $\phi_{f} \in S_{R R O}(L)$ for every $f \in L$.

Proof. Suppose that $L$ is modular and that $f, g, h \in L$. Then, since $f^{\prime} \leqq\left(g \phi_{f}\right)^{\prime}, \quad\left[\left(g \phi_{f}\right)^{\prime} \wedge h\right] \vee f^{\prime}=\left(g \phi_{f}\right)^{\prime} \wedge\left(h \vee f^{\prime}\right)$. Consequently, $\left[\left(g \phi_{f}\right)^{\prime} \wedge h\right] \phi_{f}=\left\{\left[\left(g \phi_{f}\right)^{\prime} \wedge h\right] \vee f^{\prime}\right\} \wedge f=\left(g \phi_{f}\right)^{\prime} \wedge\left(h \vee f^{\prime}\right) \wedge f=\left(g \phi_{f}\right)^{\prime} \wedge$ $f \wedge h \phi_{f}=\left(g^{\prime} \wedge f\right) \phi_{f} \wedge h \phi_{f}=g^{\prime} \wedge f \wedge h \phi_{f}=g^{\prime} \wedge h \phi_{f}$, proving that $\phi_{f} \in$ $S_{\text {RRO }}(L)$.

Conversely, suppose that $\phi_{f} \in S_{T R O}(L)$ for every $f \in L$. If $L$ were not modular, there would exist, according to Lemma 8, elements $e, f, g$ $\in L$ such that $g<e^{\prime} \phi_{f}$ and $g \vee e=f \vee e$. By Theorem 3, this would imply that $\phi_{e}, \phi_{f}$ fails to be range-closed, contradicting $\phi_{f} \in S_{T_{R O}}(L)$.

4. Conditions for the Modularity of $L$. In this section we prove our main result, namely:

THEOREM 10. Let $L$ be an orthomodular lattice. Then, the following conditions are mutually equivalent:

(i) $L$ is modular.

(ii) $L$ can be coordinatized by $a^{*}$-regular Baer *-semigroup.

(iii) $L$ can be coordinatized by a range-closed Baer *-semigroup.

Proof. If $L$ is modular, then Theorems 7 and 9 imply that $S_{r R o}(L)$ is a *-regular Baer *-semigroup coordinatizing $L$; hence, (i) $\Rightarrow$ (ii). 
In order to prove that (ii) $\Rightarrow$ (iii), we will have to recall that if $a$ and $y$ are elements of a Baer *-semigroup $S$, then $(a y)^{\prime \prime}=\left(a^{\prime \prime} y\right)^{\prime \prime}$. This was shown in the course of the proof of [2, Theorem 8, p. 654]. Now, suppose that $\mathrm{S}$ is a *-regular Baer *-semigoup coordinatizing $L$. For $b \in S$ and $g \in P^{\prime}(S) ; g \leqq b^{\prime \prime}$ and $\left(g b^{*}\right)^{\prime \prime}=\left(b^{*}\right)^{\prime \prime} \Rightarrow b^{\prime \prime}=b^{*}\left(b^{*}\right)^{-1}=\left[b^{*}\left(b^{*}\right)^{-1}\right]^{\prime \prime}$ $=\left[\left(b^{*}\right)^{\prime \prime}\left(b^{*}\right)^{-1}\right]^{\prime \prime}=\left[\left(g b^{*}\right)^{\prime \prime}\left(b^{*}\right)^{-1}\right]^{\prime \prime}=\left[g b^{*}\left(b^{*}\right)^{-1}\right]^{\prime \prime}=\left(g b^{\prime \prime}\right)^{\prime \prime}=g^{\prime \prime}=g . \quad$ Thus, any element $b \in S$ is range-closed. This proves (ii) $\Rightarrow$ (iii).

Finally, let $S$ be a range-closed $B$ aer ${ }^{*}$-semigroup, and let $e, f \in L$ $=P^{\prime}(S)$. Then, $\phi_{e} \phi_{f}=\phi_{e \rho}$ by $[2$, Theorem 8 , p. 654]. Since $S$ is range-closed, ef is range-closed in $\mathrm{S}$, so $\phi_{e f}=\phi_{e} \phi_{f}$ is range-closed in $\mathrm{S}(L)$. It follows that $\phi_{f} \in S_{T R O}(L)$ for every $f \in L$; hence, that $L$ is modular by Theorem 9 . Consequently, (iii) $\Rightarrow$ (i).

\section{REFERENCES}

1. G. Birkhoff and J. von Neumann, The logic of quantum mechanics, Ann. of Math., 37 (1936), 823-843.

2. D. J. Foulis, Baer *-semigroups, Proc. Amer. Math. Soc., 11, No. 4, (1960), 648-654.

3. I. Kaplansky, Any orthocomplemented complete modular lattice is a continuous geometry, Ann. of Math. 61 (1955), 524-541.

4. L. H. Loomis, The lattice theoretic background of the dimension theory of operator algebras, Amer. Math. Soc. Memoir No. 18, (1955).

5. G. W. Mackey, Quantum Mechanics and Hilbert Space, Herbert Ellsworth Slaught Memorial Paper No. 6, Amer. Math. Monthly, 64, No. 8, (1957), 45-57.

Wayne State University, and U. S. Naval Ordnance

Test Station, China lake, California. 



\title{
PACIFIC JOURNAL OF MATHEMATICS
}

\section{EDITORS}

\author{
Ralph S. Phillips \\ Stanford University \\ Stanford, California \\ F. H. BrownelL \\ University of Washington \\ Seattle 5 , Washington
}

A. L. Whiteman

University of Southern California

Los Angeles 7. California

L. J. PAIGE

University of California

Los Angeles 24, California

\section{ASSOCIATE EDITORS}

\author{
E. F. BECKENBACH \\ T. M. CHERRY
} \\ D. DERRY \\ M. OHTSUKA \\ UNIVERSITY OF BRITISH COLUMBIA \\ CALIFORNIA INSTITUTE OF TECHNOLOGY \\ UNIVERSITY OF CALIFORNIA \\ MONTANA STATE UNIVERSITY \\ UNIVERSITY OF NEVADA \\ NEW MEXICO STATE UNIVERSITY \\ OREGON STATE COLLEGE \\ UNIVERSITY OF OREGON \\ OSAKA UNIVERSITY \\ UNIVERSITY OF SOUTHERN CALIFORNIA
}

H. L. ROYDEN

E. SPANIER
E. G. STRAUS

F. WOLF

\section{SUPPORTING INSTITUTIONS}

\author{
STANFORD UNIVERSITY \\ UNIVERSITY OF TOKYO \\ UNIVERSITY OF UTAH \\ WASHINGTON STATE COLLEGE \\ UNIVERSITY OF WASHINGTON \\ AMERICAN MATHEMATICAL SOCIETY \\ CALIFORNIA RESEARCH CORPORATION \\ HUGHES AIRCRAFT COMPANY \\ SPACE TECHNOLOGY LABORATORIES \\ NAVAL ORDNANCE TEST STATION
}

Mathematical papers intended for publication in the Pacific Journal of Mathematics should be typewritten (double spaced), and the author should keep a complete copy. Manuscripts may be sent to any one of the four editors. All other communications to the editors should be addressed to the managing editor, L. J. Paige at the University of California, Los Angeles 24, California.

50 reprints per author of each article are furnished free of charge; additional copies may be obtained at cost in multiples of 50 .

The Pacific Journal of Mathematics is published quarterly, in March, June, September, and December. The price per volume (4 numbers) is $\$ 12.00$; single issues, $\$ 3.50$. Back numbers are available. Special price to individual faculty members of supporting institutions and to individual members of the American Mathematical Society: $\$ 4.00$ per volume; single issues, $\$ 1.25$.

Subscriptions, orders for back numbers, and changes of address should be sent to Pacific Journal of Mathematics, 103 Highland Boulevard, Berkeley 8, California.

Printed at Kokusai Bunken Insatsusha (International Academic Printing Co., Ltd.), No. 6 , 2-chome, Fujimi-cho, Chiyoda-ku, Tokyo, Japan.

PUBLISHED BY PACIFIC JOURNAL OF MATHEMATICS, A NON-PROFIT CORPORATION

The Supporting Institutions listed above contribute to the cost of publication of this Journal, but they are not owners or publishers and have no responsibility for its content or policies. 


\section{Pacific Journal of Mathematics}

\section{Vol. 11, No. $3 \quad$ BadMonth, 1961}

Errett Albert Bishop, A generalization of the Stone-Weierstrass theorem ..........

Hugh D. Brunk, Best fit to a random variable by a random variable measurable with

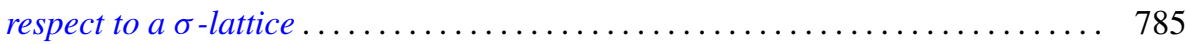

D. S. Carter, Existence of a class of steady plane gravity flows . . . . . . . . . . 803

Frank Sydney Cater, On the theory of spatial invariants ............... 821

S. Chowla, Marguerite Elizabeth Dunton and Donald John Lewis, Linear

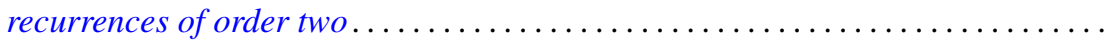

Paul Civin and Bertram Yood, The second conjugate space of a Banach algebra as

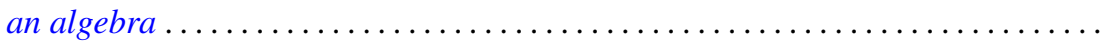

William J. Coles, Wirtinger-type integral inequalities .

Shaul Foguel, Strongly continuous Markov processes ....................

David James Foulis, Conditions for the modularity of an orthomodular lattice ...... Jerzy Górski, The Sochocki-Plemelj formula for the functions of two complex variables.

John Walker Gray, Extensions of sheaves of associative algebras by non-trivial

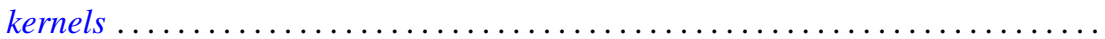

Maurice Hanan, Oscillation criteria for third-order linear differential equations .... 919 Haim Hanani and Marian Reichaw-Reichbach, Some characterizations of a class of unavoidable compact sets in the game of Banach and Mazur .............

John Grover Harvey, III, Complete holomorphs . . . . . . . . . . . . . . . . 961 Joseph Hersch, Physical interpretation and strengthing of M. Protter's method for vibrating nonhomogeneous membranes; its analogue for Schrödinger's

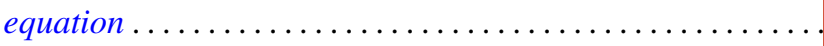

James Grady Horne, Jr., Real commutative semigroups on the plane...

Nai-Chao Hsu, The group of automorphisms of the holomorph of a group...

F. Burton Jones, The cyclic connectivity of plane continua

John Arnold Kalman, Continuity and convexity of projections and barycentric

coordinates in convex polyhedra

Samuel Karlin, Frank Proschan and Richard Eugene Barlow, Moment inequalities of

Pólya frequency functions .

Tilla Weinstein, Imbedding compact Riemann surfaces in 3-space. .

Azriel Lévy and Robert Lawson Vaught, Principles of partial reflection in the set

theories of Zermelo and Ackermann

Donald John Lewis, Two classes of Diophantine equations .....

Daniel C. Lewis, Reversible transformations . . .

Gerald Otis Losey and Hans Schneider, Group membership in rings and

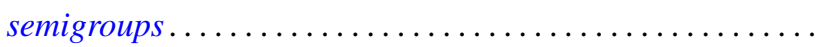

M. N. Mikhail and M. Nassif, On the difference and sum of basic sets of

polynomials.

Alex I. Rosenberg and Daniel Zelinsky, Automorphisms of separable algebras .

Robert Steinberg, Automorphisms of classical Lie algebras .... 